\title{
Phlebologie
}

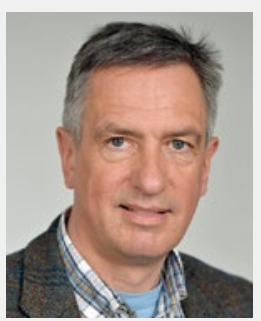

Prof. Dr. Achim Mumme, Bochum

\section{Operative Phlebologie: Vielfalt in der Phlebologie}

„Die moderne Phlebologie glänzt durch eine nie gekannte Methodenvielfalt.“ So oder ähnlich klingt es immer wieder auf unseren nationalen und internationalen Tagungen. Es wird eine „Krampfadernbehandlung à la carte“ proklamiert, bei der die verschiedenen Therapieoptionen - operative, endovenöse oder sklerosierende - entsprechend ihrer jeweiligen Vor- oder Nachteile quasi maßgeschneidert zum Einsatz kommen. Diese vollmundigen Empfehlungen sind aber, bei genauer Betrachtung, nicht viel mehr als ein Lippenbekenntnis.

Schließlich richtet sich die Auswahl der Behandlungsmethoden nicht allein nach medizinischen, sondern auch nach wirtschaftlichen Kriterien. Dies gilt vor allem dann, wenn verschiedene therapeutische Alternativen zur Verfügung stehen. In einer solchen Situation ist bei der Methodenauswahl der Anreiz gegeben, die wirtschaftlich interessanteste Verfahrensweise zu bevorzugen. Weniger lukrative Verfahren kommen dann ins Hintertreffen bis sie schließlich kaum noch verfügbar sind. Vom gewünschten À-la-carteMenü bleibt dann am Ende nur noch ein singuläres Einheitsgericht über.

Ein gutes Beispiel hierfür gibt es im US-amerikanischen Raum, wo die endovenöse Therapie die alternativen Verfahren inzwischen weitestgehend verdrängt hat. Entsprechend einer jüngst publizierten Analyse [1] ist die dortige Mengenausdehnung endovenöser Verfahren nicht zuletzt auf wirtschaftliche Fehlanreize zurückzuführen. Die (nicht nur in den USA) im Vergleich zu endovenösen Verfahren schlecht vergütete Varizenoperation hatte gegenüber der wesentlich lukrativeren und massiv von der Industrie beworbenen [2] Behandlungsalternative von vornherein keine wirkliche Chance. Wenn wir tatsächlich die Methodenvielfalt in der Phlebologie erhalten möchten, ist es aber notwendig, wirtschaftliche Fehlanreize zu vermeiden und für eine auskömmliche Erstattung aller sinnvollen Therapieoptionen zu sorgen. Vielleicht hat auch das übliche FreundFeind-Denken bisher die Diskussion unnötig erschwert. Wir brauchen einen Konsens innerhalb der Phlebologie, dass für uns die Vielfalt der Methoden eine hohe Präferenz hat.

Vielfalt ist auch ein Thema innerhalb der operativen Phlebologie, die sich längst nicht mehr nur mit der ablativen Therapie von Krampfadern beschäftigt. Unter dem Einfluss des hochauflösenden Ultraschalls konnten sich, alternativ zum Stripping, venenerhaltende Konzepte entwickeln, die auf die Reparatur des Klappenapparates abzielen.

In einer Originalarbeit aus der Bochumer Arbeitsgruppe stellen Bruno Geier und Mitarbeiter die 10-Jahres-Ergebnisse einer prospektiven Studie dar, in der Venenklappenrekonstruktionen mit dem Verfahren der extraluminalen Valvuloplastie erfolgten.

Einen anderen Weg zur Erhaltung der Stammvene beschreibt Erika Mendoza. Sie gibt uns einen Überblick über die Verfahrensweise bei der CHIVA-Methode, die in erster Linie auf der Ausschaltung von Rezirkulationskreisläufen basiert.

Achim Hermanns hat durch seine besondere Expertise in der Shave-Therapie ein internationales Renommee erworben. Seine Übersichtsarbeit über die Standards bei der ope- 
rativen Behandlung des Ulcus cruris beleuchtet ein besonders wichtiges Feld der operativen Phlebologie: Es geht um die Behandlung unserer schwierigsten Fälle.

Schließlich runden Guido Bruning und Johanna Buhr das Themenheft mit ihrem Beitrag „Varizenchirurgie: Belastender als andere Verfahren?“ ab. Mit ihrem Statement aus dem Blickwinkel eines großen Venenzentrums unternehmen sie den Versuch einer Standortbestimmung der Varizenoperation innerhalb des phlebologischen „Menu à la carte“.

Viel Freude bei der Lektüre wünscht Ihnen

Ihr

Achim Mumme

Literatur

[1] Baber M], Sedrakyan A, Connolly PH et al. Impact of provider characteristics on use of endovenous ablation procedures in Medicare beneficiaries. J Vasc Surg Venous Lymphat Disord 2019; 7 (2):203-209

[2] Bosanquet DC, Twine CP. The Endovenous Literature: A Perfect Storm of Limited Effectiveness Data, Rapid Technological Evolution and Potential Conflict of Interest. Eur J Vasc Endovasc Surg 2017; 54: 771 